\title{
Condiciones de seguridad percibidas por cuidadores familiares en atención pediátrica
}

\author{
Safety conditions perceived by family caregivers in pediatric care \\ Condições de segurança percebidas pelos cuidadores familiares no atendimento pediátrico
}

\author{
Elizabeth Romero-Massa* \\ Angely Carrera-Hooker ${ }^{* *}$ \\ Danna Garcia-Martínez ${ }^{* * *}$
}

\begin{abstract}
Autor de correspondencia
* $\bowtie$ Enfermera. Especialista en Enfermería Cardiológica UNAM. Magister en Enfermería. Profesora titular, Facultad de Enfermería, Universidad de Cartagena. Correo: eromerom@unicartagena.edu.co. Orcid: https:// orcid.org/0000-0001-5504-7410. Cartagena, Colombia.

** Enfermera. Universidad de Cartagena. Enfermera de turno, Hospital de San Andrés, Archipiélago de San Andrés, Correo: carrerahangely@hotmail.com Orcid: https:// orcid.org/0000-0003-2668-4925. Cartagena, Colombia.

${ }^{* * *}$ Enfermera. Universidad de Cartagena. Enfermera de turno, Hospital Universitario del Caribe, Cartagena. Correo: dannaaries2110@ hotmail.com. Orcid: https://orcid.org/00000001-9810-2547. Cartagena, Colombia.
\end{abstract}

Este es un artículo bajo la licencia CC BY

(https://creativecommons.org/

licenses $/$ by $/ 4.0 /$ ) (c) ()

\section{Resumen}

Objetivo: Identificar la percepción de los cuidadores sobre condiciones de seguridad en la atención en un hospital pediátrico. Materiales y Métodos: Estudio descriptivo. Por muestreo probabilístico se seleccionaron y participaron 163 cuidadores de niños hospitalizados. Se aplican la encuesta de caracterización y la de condiciones de seguridad del entorno hospitalario versión cuidador, con alfa de Cronbach de 0.97. Mediante estadística descriptiva se expresan los datos Resultados: Los cuidadores en el 51,5\% tenían edades entre 18-29 años, en su mayoría del sexo femenino y madres, correspondiendo al $95 \%$ y $85,9 \%$ respectivamente. Percibieron seguridad en la hospitalización del niño el $86 \%$; ocurrencia de riesgos por flebitis el 17\%; reacciones adversas a medicamentos 8,6\%; confusión de pacientes 7,4\%. En menor grado caídas, infecciones y ulceras por presión en el 2,4\%. En mayor proporción se reportaron en riesgo: $21,5 \%$ flebitis, $19 \%$ caídas, $11 \%$ infecciones, $10 \%$ reacciones adversas medicamentosas y $8,6 \%$ confusión de pacientes. Para los cuidadores, al $60.2 \%$ les explican los procedimientos, la atención es oportuna para el 59\%, la comunicación es clara para el 70\%, están alertas para detectar posibles riesgos el $58.3 \%$, siguen recomendaciones dadas el 75.5\%, confían en los profesionales el $70.5 \%$, manejan situaciones propias de la hospitalización el 63.2\%. Conclusiones: Los cuidadores se sentían seguros, conocían y se mantenían informados sobre los riesgos y la razón de la hospitalización del niño. Por otra parte, su percepción sobre las condiciones de seguridad y la atención, permitió determinar que existe confianza en el profesional que atiende en la hospitalización pediátrica. Se encontró que la flebitis, reacciones a medicamentos y confusión de pacientes fueron los riesgos de mayor ocurrencia.

Palabras clave: Percepción, cuidadores, cuidado seguro, seguridad del paciente. Fuente: DeCS 


\begin{abstract}
Objective: Identify the perception of caregivers about the safety conditions in pediatric care in the children's hospital. Materials and methods: Descriptive study; 163 caregivers of hospitalized children participated and were selected through probabilistic sampling, the characterization interview and the safety conditions of the hospital environment (caregiver version) interview were applied, with a Cronbach's alpha of 0.97, the data is expressed through descriptive statistics. Results: $51.5 \%$ of the caregivers correspond to an age range between 18-29 years, the majority are females $(95 \%)$ and mothers (85.9\%). 86\% perceived safety in the hospitalization of the child, $17 \%$ risk by phlebitis, $8.6 \%$ adverse reactions to the medication and $7.4 \%$ confusion in the patient, while, in a lesser degree, $2.4 \%$ perceived falls, infections and pressure sores. In a greater proportion, the reported risk of phlebitis was $21.5 \%$, $19 \%$ falls, $11 \%$ infections, $10 \%$ adverse reactions to medication and $8.6 \%$ confusion in the patient. For the caregivers, $60.2 \%$ explain the procedures, $59 \%$ consider prompt medical care, communication is clear for $70 \%, 58.3 \%$ are on the alert of detecting possible risks, $75.5 \%$ follows the given recommendations, $70.5 \%$ trust the professionals and $63.2 \%$ handles specific situations from the hospitalization. Conclusions: The caregivers felt safe, knew and kept informed about the risks and the reason for the hospitalization of the child; also, their perception about the safety conditions allowed to identify trust in the professional that assists in the pediatric hospitalization. It was found that phlebitis, reactions to medication and confusion in the patients were the risks with greater occurrence.
\end{abstract}

Keywords: Perception, caregivers, safe care, patient safety.

\title{
Resumo
}

Objetivo: Identificar a percepção dos cuidadores sobre a segurança do atendimento num hospital pediátrico Materiais e Métodos: Estudo descritivo, selecionou por amostragem probabilística 163 cuidadores de crianças internadas. Aplicou-se um instrumento de caraterização e de avaliação de condições segurança do ambiente hospitalar versão cuidador, com alpha de Cronbach de 0,97. Empregou-se estatística descritiva para apresentar os resultados. Resultados: A idade dos cuidadores esteve 51,5\% entre os 18-29 anos, a maioria foram do sexo feminino (95\%), sendo predominantemente as mães da criança internada $(85,9 \%)$. A percepção de segurança foi positiva $(86 \%)$, entretanto, apresentaram-se eventos como flebites $(17 \%)$, reações adversas aos medicamentos $(8,6 \%)$, e confusão (erro) de paciente $(7.4 \%)$. Em menores proporções observaram-se quedas $(2,4 \%)$ infecções e lesões por pressão. As maiores ameaças reportadas foram risco de apresentar flebites $(21.5 \%)$, quedas $(19 \%)$, infecções $(11 \%)$, reações adversas aos medicamentos $(10 \%)$ e confusão de paciente (8.6\%). Para os cuidadores, $60,2 \%$ recebeu explicações dos procedimentos, $59 \%$ qualificou o atendimento como oportuno, a comunicação foi clara (70\%), está atento na detecção de possíveis riscos $(58,3 \%)$, segue as recomendações que lhe foram dadas $(75,5 \%)$, confia nos profissionais $(70,5 \%)$ e consegue contornar as situações próprias da internação (63,2\%). Conclusões: Os cuidadores sentiam-se seguros, conheciam e se mantinham informados dos riscos e a razão da internação das crianças; além disso, a sua percepção sobre as condições de segurança no atendimento, permitiram identificar confiança no profissional que atendia na enfermaria da pediatria. Encontrou-se que a flebite, as reações aos medicamentos e o erro do paciente foram os riscos de maior ocorrência.

Palavras-chave: Percepção, cuidadores, segurança do paciente

\section{Introducción}

La enfermería por permanecer de forma directa y constante desempeña un papel decisivo en la promoción de la seguridad del paciente. Si este cuidado no se realiza con calidad puede generar errores y poner en peligro la seguridad del cuidado (1).

Teniendo en cuenta las características anteriores sobre la enfermería, es importante señalar que el cumplimiento de la normatividad vigente que contribuye a garantizar la actuación de los profesionales de la salud, así como de las instituciones, en todo lo relacionado con la seguridad del paciente, está indicado en la resolución 2003 de habilitación, que exige cumplir con una serie de herramientas para implementarla, siguiendo los lineamientos y las guías del Ministerio de Protección Social $(2,3)$.

La Seguridad del paciente es el conjunto de elementos estructurales, procesos, instrumentos y metodologías basadas en evidencias científicamente probadas que 
ISSN-PRINT

$1794-9831$

E-ISSN 2322-7028

Vol. 16 No. 3

Sep - Dic 2019

Cúcuta, Colombia propenden a minimizar el riesgo de sufrir un evento adverso en el proceso de atención de la salud o de mitigar sus consecuencias (4).

Por otra parte, el interés por eliminar o disminuir los riesgos, así como los efectos adversos relacionados con la atención en salud es desde hace varias décadas una prioridad. Por esta razón, la Organización Mundial de la Salud, la Organización Panamericana de la Salud y otros organismos internacionales han planteado y estudiado nuevas acciones para que se puedan controlar y evitar los posibles efectos adversos en la práctica asistencial (5).

Colombia impulsa una Política de Seguridad del Paciente, liderada por el Sistema Obligatorio de Garantía de Calidad de la Atención en Salud, cuyo objetivo "es prevenir la ocurrencia de situaciones que afecten la seguridad del paciente, reducir y de ser posible, eliminar la ocurrencia de eventos adversos para contar con instituciones seguras" (6).

Cabe destacar que si se promueve en el niño, la familia y los profesionales de la salud acciones que contribuyan a la seguridad en la atención, se constituyen en agentes activos en la promoción de acciones haciendo que los procedimientos sean más seguros, permitiéndoles ser capaces de comprender y participar del cuidado (7).

En el ámbito hospitalario se encontraron estudios que se relacionan específicamente con condiciones de seguridad en hospitales pediátricos. Delgadillo (2014) señala que en relación con la satisfacción de la madre del recién nacido hospitalizado respecto al cuidado del profesional de enfermería, el $61 \%$ tiene satisfacción media. En este sentido la madre percibe que sus cuidados influyeron positivamente en la salud de su bebé, por lo general permaneció a su servicio, casi siempre mostró seguridad cuando brindó sus cuidados y frecuentemente se lava las manos antes y después de los procedimientos. En contraste, las madres en un $21 \%$ percibieron baja satisfacción con respecto a la atención por parte de la enfermería, al mostrar desinterés en el estado de salud de su bebé. Contrariamente, el 18\% restante tuvo una satisfacción alta, debido a que su bebé siempre se mantuvo protegido por las corrientes de aire y los insumos que le ofrecieron fueron suficientes (8).

Se debe precisar que la enfermera afronta situaciones que representan riesgos de prácticas inseguras en el cuidado de los niños. Como consecuencia existe una gran probabilidad de identificación del error, debido a la presencia de acontecimientos que ponen en peligro la seguridad tales como: fallo en la comunicación oral o escrita entre profesionales del equipo y entre profesional y paciente; y, fallo en la terapéutica medicamentosa, sea en la prescripción, preparación, administración. Del mismo modo, estos errores se presentan debido a eventos adversos causados por fallos en la realización de procedimientos de salud, como flebitis, úlceras por presión, infecciones, transfusión sanguínea, procedimiento peri operatorios, hemodiálisis, terapia del calor y factores humanos $(9,10)$.

Igualmente, existen factores que comprometen la calidad del cuidado y la enfermería tiene un papel fundamental en garantizar la calidad de la asistencia prestada (11).

Por otro lado, la disciplina exige de la enfermería acciones seguras a través de sus prácticas de cuidado a los niños hospitalizados y a sus cuidadores en la promoción de políticas de prevención de errores, para alcanzar la adecuada calidad asistencial y asegurar el cumplimiento de la normatividad vigente.

Es importante destacar, que se hizo necesario indagar sobre la percepción de los cuidadores de niños hospitalizados, así como de las prácticas seguras de salud que reciben por parte de la enfermería, con el objetivo de ofrecer mejores prácticas de cuidado y compartir los resultados promoviendo un desarrollo de cuidado seguro pediátrico.

Por este motivo, en el logro de los procesos de atención en salud, la percepción de los cuidadores se constituirá en un elemento para responder a las necesidades de cada uno de ellos en la búsqueda de una mejor gestión del cuidado seguro.

\section{Objetivo General}

- Identificar la percepción de los cuidadores sobre las condiciones de seguridad en la atención en un hospital pediátrico de Cartagena.

\section{Objetivos Específicos}

- Describir las características sociodemográficas de los cuidadores de niños en un hospital pediátrico de Cartagena. 
- Describir las percepciones de los cuidadores de niños hospitalizados sobre las condiciones de seguridad en la atención hospitalaria, teniendo en cuenta los siguientes aspectos: conocimiento sobre riesgos y percepción de riesgo ante eventos adversos, percepción de procedimientos y/o pautas de prevención de riesgos y complicaciones

\section{Materiales y Métodos}

Estudio descriptivo y transversal. La población estuvo compuesta por los participantes que permanecieron al cuidado de su familiar hospitalizado en cada una de las 72 camas censables en un hospital pediátrico de Cartagena, durante un trimestre del año 2017. La muestra se obtuvo mediante cálculo de población finita con un nivel de confianza del $95 \%$, una prevalencia esperada del $50 \%$ y un error del $6 \%$. Se consiguió una muestra de 163 cuidadores, por muestreo probabilístico aleatorio simple, que cumplieron con los siguientes criterios de inclusión: ser cuidador familiar, mayor de 18 años, mentalmente competente y que deseara participar de manera voluntaria en el estudio, previa firma del consentimiento informado. Actividad que se realizó en los horarios de visita de cada cuidador en las salas de los servicios de urgencias, quirófanos, hospitalización, UCIN, UCI pediátrica, Unidad de Cuidados Cardiovasculares y Unidad Oncológica.

Para recolectar la información se emplearon dos instrumentos, uno es el de caracterización del cuidador familiar del Grupo de Cuidado al paciente crónico y su familia de la Universidad Nacional. Este instrumento incluye la caracterización de los cuidadores familiares de personas con ECNT, con obtención de permisos para su utilización, porque hace parte del Nodo de la red latinoamericana del cuidado al pa- ciente crónico y su familia. Incluye datos sociodemográficos como: sexo, edad, estrato, relación con el cuidador, tiempo que lleva con la enfermedad crónica, nivel de religiosidad, escolaridad (12).

Otro Instrumento utilizado fue el GCPC-UN-C que evalúa la percepción de los cuidadores sobre condiciones de seguridad del entorno hospitalario; cuenta con pruebas de validez y su confiabilidad fue de un alfa de Cronbach de 0.97 (13).

El GCPC-UN-C consta de 4 dimensiones y 37 ítems que se miden con preguntas dicotómicas de si y no tipo likert y una pregunta abierta que mide la percepción global de seguridad en el hospital con 1 ítem. Para realizar la interpretación de los resultados se tendrá en cuenta valores de $100 \%$ para estas respuestas.

Dimensiones:

1. Percepción de conocimiento sobre riesgos: 7 ítems y preguntas dicotómicas

2. Percepción de Ocurrencia de riesgo de eventos adversos: 8 ítems, preguntas dicotómicas para ocurrencia del riesgo y 8 si ha estado en riesgo de ocurrir.

3. Percepción de pautas de prevención: 5 ítems

4. Percepción de comportamientos frente al riesgo:8 ítems

Percepción global de seguridad: 1 ítem

Para las dimensiones 3 y 4, la interpretación de los resultados de las respuestas tipo likert van de 0 a 4 , donde se suman los resultados en un puntaje total de rango por cada dimensión; este resultado clasificó la percepción del cuidador sobre cuidado seguro en: nunca, algunas veces, casi siempre y siempre, de acuerdo a los puntajes (ver tabla 1).

Tabla 1. Interpretación de resultados de las dimensiones sobre cuidado seguro

\begin{tabular}{lllll}
\hline \multicolumn{1}{c}{ Dimensiones } & Nunca & Algunas veces & Casi siempre & Siempre \\
\hline Pautas de Prevención & 0 a 4 & $>4$ a 16 & $>16$ a 32 & $>32$ \\
Comportamiento frente al riesgo & 0 a 4 & $>4$ a 16 & $>16$ a 32 & $>32$ \\
\hline
\end{tabular}

Fuente: Elaboración Propia

Una vez obtenidos los datos fueron registrados en un formato previamente diseñado para este propósito específico en Excel y compatible con el Programa SPSS versión 17.0. Los resultados de la investigación se presentaron con estadística descriptiva y se tuvieron en cuenta las frecuencias y porcentajes de las opciones siempre y casi siempre. 
ISSN-PRINT

1794-9831

E-ISSN 2322-7028

Vol. 16 No. 3

Sep - Dic 2019

Cúcuta, Colombia
Se contó con los avales del comité de ética institucional de la Universidad de Cartagena y de la Institución Hospitalaria. De conformidad con lo establecido en la resolución 008430 de 1993 (14), se consideró de bajo riesgo, basado en los principios de autonomía, justicia, beneficencia y no maleficencia.

\section{Resultados}

Participaron 163 cuidadores de niños hospitalizados. Se encontró que el 51,5\% tenían entre 18 a 29 años, seguido de quienes contaban entre los 30 a 59 años en un $48,5 \%$. El género que predominó fue el femenino en un $95 \%$. Se observó que $38,6 \%$ estudiaron secundaria completa, seguido de secundaria incompleta con el $22,1 \%$. Se dedica a las tareas del hogar el $65 \%$, mientras que el $25,1 \%$ trabaja. El $85.9 \%$ son madres, seguido del 5,5\% que son abuelas, mientras que el $4,3 \%$ son tías y aplica el mismo porcentaje para padres. Forman su hogar en unión libre el 57,7\%. El $24 \%$ son solteros, los casados representan el $16,6 \%$. Los cuidadores en estrato de nivel muy bajo correspondió al 84,7\%. Conocimiento frente al riesgo durante la Hospitalización.

Los cuidadores en el 100\% conocían el motivo de la hospitalización y la razón de la misma. Percibieron que su hijo estaba expuesto a riesgos en el $62 \%$. Se pudo establecer que el 51\% desconocía cómo ayudar a prevenir riesgos. Respecto a la actuación ante el riesgo, el 64.4\% lo ignoraban, La población encuestada en un $63.2 \%$ no conocía los derechos y deberes del paciente (Tabla 2).

Tabla 2. Conocimientos del cuidador frente al riesgo en la hospitalización pediátrica (2017)

\begin{tabular}{lcccc}
\hline Conocimiento & \multicolumn{2}{c}{$\mathrm{Si}$} & \multicolumn{2}{c}{ No } \\
\hline Exposición a riesgos & $\mathrm{Fr}$ & & $\mathrm{Fr}$ & \\
& 101 & $62 \%$ & 62 & $38 \%$ \\
Ayuda a prevenir riesgos & 80 & $49 \%$ & 83 & $51 \%$ \\
Actuación ante el riesgo & 58 & $35,6 \%$ & 105 & $64,4 \%$ \\
Deberes y derechos de los pacientes & 60 & $36,8 \%$ & 103 & $63,2 \%$ \\
\hline
\end{tabular}

Fuente: Encuestas aplicadas en el Hospital.

Percepción de riesgo materializado ocurrido o en riesgo de ocurrencia.

En los hallazgos en cuanto a las caídas, el 5\% aseveró que su familiar las ha sufrido, mientras que el $19 \%$ aseguró que han estado en riesgo. En relación a las úlceras por presión para los cuidadores ocurrieron en el $2.4 \%$. Un $8.6 \%$ presentaron reacción a la administración de medicamentos en los niños (Tabla $3)$. Respecto a la administración de medicamentos para el 13,5\% de cuidadores encontró la ocurrencia en horarios no establecidos. El retiro accidental de dispositivos invasivos lo reportaron el 5\% de los participantes y en riesgo de retiro el 5,5\%. Los cuidadores han percibido la ocurrencia de infecciones en el $2.4 \%$; observaron ocurrencia de flebitis un $17 \%$. El riesgo de ocurrencia en niños de flebitis fue observado en el $21,5 \%$ de cuidadores. De los encuestados el $7.4 \%$ afirmó que a su hijo lo han confundido con otro paciente (Tabla 3). 
Tabla 3. Riesgo materializado ocurrido o en riesgo de ocurrencia: caídas, úlceras por presión, administración de medicamentos, retiro de dispositivos, infecciones y confusión de pacientes en un Hospital pediátrico (2017).

\begin{tabular}{|c|c|c|c|c|}
\hline \multirow{2}{*}{$\begin{array}{l}\text { Riesgo ocurrido o en riesgo } \\
\text { Ocurrencia de caídas }\end{array}$} & \multicolumn{2}{|c|}{$\mathbf{S i}$} & \multicolumn{2}{|c|}{ No } \\
\hline & 7 & $5 \%$ & 156 & $95 \%$ \\
\hline Riesgo de caídas & 31 & $19 \%$ & 132 & $81 \%$ \\
\hline Ocurrencia de úlceras por presión & 4 & $2,4 \%$ & 159 & $97,6 \%$ \\
\hline Riesgo de úlceras por presión & 9 & $5,5 \%$ & 154 & $94,5 \%$ \\
\hline Ocurrencia administración de medicamentos en horario no establecido & 22 & $13,5 \%$ & 141 & $86,5 \%$ \\
\hline Riesgo de administración de medicamentos en horario no establecido & 27 & $16,6 \%$ & 136 & 83,4 \\
\hline Ocurrencia de reacción a la administración de medicamentos & 14 & 8,6 & 149 & $91,4 \%$ \\
\hline Riesgo de reacción a la administración de medicamentos & 16 & $10 \%$ & 147 & $90 \%$ \\
\hline Ocurrencia retiro accidental dispositivos & 7 & $5 \%$ & 156 & $95 \%$ \\
\hline Riesgo retiro accidental dispositivos & 9 & $5,5 \%$ & 154 & 94 \\
\hline Ocurrencia de infecciones & 4 & $2,4 \%$ & 159 & $97,6 \%$ \\
\hline Riesgo de infecciones & 18 & $11 \%$ & 145 & $89 \%$ \\
\hline Ocurrencia de flebitis & 28 & $17 \%$ & 135 & $83 \%$ \\
\hline Riesgo de flebitis & 35 & $21,5 \%$ & 128 & $78,5 \%$ \\
\hline Ocurrencia de confusión de pacientes & 12 & $7,4 \%$ & 151 & $92,6 \%$ \\
\hline riesgo de confusión de pacientes & 14 & $8,6 \%$ & 149 & $91,4 \%$ \\
\hline
\end{tabular}

Fuente: Encuestas aplicadas en el Hospital.

\section{Percepción de procedimientos y/o pautas de prevención de riesgos y complicaciones.}

Los cuidadores percibieron siempre en un $60.2 \%$ y casi siempre el $19,6 \%$ la explicación sobre reglas y procedimientos de seguridad ofrecida por el equipo de salud durante la hospitalización del niño (tabla 4). De igual manera los cuidadores indicaron que siempre acuden oportunamente ante los llamados al personal de salud, en un $59 \%$ y casi siempre $25,1 \%$ (Tabla 4 ).

la identificación por su nombre de parte de los profesionales lo observaron los cuidadores siempre en el $73,7 \%$ y casi siempre el $15,3 \%$. Por otra parte, señalaron que siempre existió una comunicación clara con el personal de salud en el 70\% (Tabla 4).

Tabla 4. Percepción de procedimientos y/o pautas de prevención de riesgos y complicaciones (2017).

\begin{tabular}{|c|c|c|c|c|c|c|c|c|}
\hline \multirow{2}{*}{$\begin{array}{l}\text { Pautas } \\
\text { Explicación de reglas y procedimientos de seguridad }\end{array}$} & \multicolumn{2}{|c|}{ Nunca } & \multicolumn{2}{|c|}{ Algunas veces } & \multicolumn{2}{|c|}{ Casi siempre } & \multicolumn{2}{|c|}{ Siempre } \\
\hline & 11 & $6,7 \%$ & 22 & $13,5 \%$ & 32 & $19,6 \%$ & 98 & $60,2 \%$ \\
\hline El personal de salud acude oportunamente a llamados & 2 & $1,2 \%$ & 24 & $14,7 \%$ & 41 & $25,1 \%$ & 96 & $59 \%$ \\
\hline $\begin{array}{l}\text { El personal de salud identifica o llama por su nombre a } \\
\text { su familiar }\end{array}$ & 0 & $0 \%$ & 18 & $11 \%$ & 25 & $15,3 \%$ & 120 & $73,7 \%$ \\
\hline $\begin{array}{l}\text { Comunicación es plena, clara y confidente con el } \\
\text { personal de salud }\end{array}$ & 0 & $0 \%$ & 24 & $14,7 \%$ & 25 & $15,3 \%$ & 114 & $70 \%$ \\
\hline $\begin{array}{l}\text { Recibe información sobre riesgos y complicaciones de } \\
\text { los tratamientos y procedimientos }\end{array}$ & 20 & $12,3 \%$ & 19 & $11,6 \%$ & 40 & $24,6 \%$ & 84 & $51,5 \%$ \\
\hline
\end{tabular}

Fuente: Encuestas aplicadas en el Hospital.

Los cuidadores familiares siempre y casi siempre recibían información sobre riesgos, complicaciones y procedimientos realizados durante la hospitalización en el $51,5 \%$ y el $24,6 \%$ respectivamente (Tabla 4 ). 
ISSN-PRINT

$1794-9831$

E-ISSN 2322-7028

Vol. 16 No. 3

Sep - Dic 2019

Cúcuta, Colombia
Comportamiento frente al riesgo en la Hospitalización

Los cuidadores respondieron en el $65 \%$ que siempre al llegar a un lugar nuevo lo observaban con atención. En este sentido, también informaron que siempre estaban atentos para detectar posibles riesgos en el espacio en un 58,3\%. En cuanto a buscar apoyo en la detección de un posible riesgo, los cuidadores afirmaron que lo hacen en un $65,6 \%$ y $17,3 \%$ responde casi siempre. Igualmente, un $61,3 \%$ detectaron un posible riesgo y hacían algo para minimizarlo. Los cuidadores siguieron las recomendaciones de seguri- dad formuladas por el personal de salud en un $75,5 \%$. También sintieron confianza en los profesionales que los atendieron en la hospitalización en un 70,5\% (tabla 5). La mayoría de los cuidadores manejó condiciones y eventualidades propias de la hospitalización en un $63,2 \%$, seguido de casi siempre en un $29,4 \%$. Del mismo modo, el 72, 4\% percibió que el personal que lo atiende cumple las medidas para prevenir los riesgos, seguido de casi siempre con un 15,9\% (Tabla 5). En relación a la percepción global de seguridad, la mayoría de los cuidadores piensan que su familiar está seguro durante la hospitalización en un $86 \%$.

Tabla 5. Comportamiento frente al riesgo.

\begin{tabular}{|c|c|c|c|c|c|c|c|c|}
\hline \multirow{2}{*}{$\begin{array}{l}\text { Comportamientos } \\
\text { Cuándo usted llega a un lugar nuevo ¿lo observan con atención? }\end{array}$} & \multicolumn{2}{|c|}{ Nunca } & \multicolumn{2}{|c|}{ Algunas veces } & \multicolumn{2}{|c|}{ Casi siempre } & \multicolumn{2}{|c|}{ Siempre } \\
\hline & 0 & $0 \%$ & 19 & $11,6 \%$ & 38 & $23,4 \%$ & 106 & $65 \%$ \\
\hline Está atento para detectar posibles riesgos en el espacio & 0 & $0 \%$ & 32 & $19,6 \%$ & 36 & $22,1 \%$ & 95 & $58,3 \%$ \\
\hline Al detectar un posible riesgo, busca apoyo & 3 & $1,8 \%$ & 25 & $15,3 \%$ & 28 & $17,3 \%$ & 107 & $65,6 \%$ \\
\hline Al detectar un posible riesgo, hace algo para minimizarlo & 2 & $1,2 \%$ & 36 & $22,2 \%$ & 25 & $15,3 \%$ & 100 & $61,3 \%$ \\
\hline Sigue las recomendaciones de seguridad formuladas & 0 & $0 \%$ & 5 & $3 \%$ & 35 & $21,5 \%$ & 123 & $75,5 \%$ \\
\hline $\begin{array}{l}\text { Siente confianza en los profesionales que lo atienden en la } \\
\text { hospitalización }\end{array}$ & 1 & $0,6 \%$ & 19 & $11,7 \%$ & 28 & $17,2 \%$ & 115 & $70,5 \%$ \\
\hline Capacidad de manejar las condiciones y eventualidades & 0 & $0 \%$ & 12 & $7,4 \%$ & 48 & $29,4 \%$ & 103 & $63,2 \%$ \\
\hline El personal de salud cumple las medidas para prevenir los riesgos & 6 & $3,7 \%$ & 13 & $8 \%$ & 26 & $15,9 \%$ & 118 & $72,4 \%$ \\
\hline
\end{tabular}

Fuente: Encuestas aplicadas en el Hospital.

\section{Discusión}

En el presente estudio se indagó sobre la percepción que tiene el cuidador sobre cuidado seguro durante la hospitalización de niños, que de manera global fue afirmativa en $86 \%$ de los participantes,

Lima (1) en lo que se refiere a las prácticas seguras en el cuidado de Enfermería infiere que deben ser impulsadas para detener los fallos en la atención, promover ambientes de cuidado centrado en el paciente y la familia, favorecer las relaciones interpersonales, mejorar el sistema de prestación de la atención, con promoción de políticas de prevención de errores y de seguridad y calidad del proceso asistencial (1).

Por su parte, Vargas et al. (15) concluyen que en cuanto a la percepción de seguridad en el entorno hospitalario, diversas investigaciones han demostrado que existe una asociación positiva entre el grado de conocimiento de los pacientes sobre su condición de salud y un mejor seguimiento de los tratamientos y recomendaciones que realiza el equipo que lo atiende.

Este hallazgo es relevante y debe ser considerado por los directivos de enfermería de las instituciones de salud. De ahí, que se requiera la implementación de estrategias dirigidas a mejorar aspectos como el involucramiento de la familia en la atención.

Con respecto a las características sociodemográficas, en el presente estudio se observó el predomino del cuidador de género femenino, de ocupación hogar y el parentesco con la persona cuidada fue $85,9 \%$ madres, con estudios de secundaria completa en el $38,6 \%$ y en nivel socioeconómico muy bajo el $84,7 \%$. Similares hallazgos encontraron en su estudio Melo et al. (16) en relación a los acompañantes del niño, quienes fueron madres en el $82 \%$ y apenas un $1 \%$ de otros acompañantes. Además predominó el nivel de instrucción de enseñanza básica completa o secundaria incompleta $63 \%$, con un nivel socioeconómico de 
Graffar razonable/bueno (82\%). De igual modo Alvarado y Achury (17) encuentran que el cuidado de los niños hospitalizados con cuidados de sondas por familiares recae en madres entre 25 y 46 años.

Sobre el conocimiento frente al riesgo durante la hospitalización, los cuidadores en su totalidad conocían el motivo de la hospitalización de su familiar y además sabían que durante esta podían estar expuestos a riesgos, lo que coincide con lo expresado por Vargas et al. (15) quienes frente a las categorías de percepción de riesgo, encuentran que solamente la mitad conoce los riesgos a los que está expuesto y la forma de actuar para prevenirlos.

Por otra parte, el $63,2 \%$ de los cuidadores desconocían los derechos y deberes de los pacientes, a diferencia de los hallazgos de Vargas et al. (15), quienes encontraron que solo la mitad de pacientes reportó conocer sus deberes y derechos durante la hospitalización. Por este motivo, las instituciones se ven obligadas a informar a pacientes y acompañantes sobre los derechos y deberes durante su estancia hospitalaria. De ahí que los profesionales de la enfermería al liderar y coordinar la atención brindada por su equipo de trabajo, tiene el control directo sobre todos los factores que intervienen en la atención segura en salud, lo que implica que los cuidadores tengan conocimiento de los derechos. Con frecuencia este punto se omite, lo que significa que se debe trabajar en la institución para mejorar y brindar la información oportuna y reforzar la atención en salud.

También se encontró que la flebitis, las reacciones a medicamentos y confusión de pacientes fueron los riesgos de mayor ocurrencia percibidos por los cuidadores. Por su parte Alvarado y Achury (17) expresan que los eventos adversos que se presentan con mayor frecuencia en el paciente pediátrico son la infección, complicaciones de catéteres y medicamentos. Los cuidados proporcionados en la atención en salud a los niños lleva tácito el riesgo de generar eventos adversos que pueden ocasionar complicaciones, secuelas o incluso la muerte. Se debe indicar, que Parra et al. (18) en su estudio al caracterizar los EA e incidentes encuentran que el principal origen es el relacionado con Infecciones Asociadas a la Atención en Salud con $46 \%$ y errores en la medicación con $25,4 \%$. En este aspecto los cuidadores del presente estudio informan que solo el $2.4 \%$ ha sufrido infecciones y un gran porcentaje que no han estado en riesgo,
Cabe destacar, que los cuidadores percibieron respecto al tema de ocurrencia de riesgo de eventos adversos durante la hospitalización en niños que el 5\% sufrió caídas y el 19\% han estado en riesgo de que ocurran. En este sentido, Riquelme et al. (19) encontraron la ocurrencia de eventos adversos en el 15,7\% de los niños y en el $6,1 \%$ se registró de dos o más EA. En orden de importancia los EA reportados fueron en administración de medicamentos, caídas y golpes. Para la enfermería la prevención de caídas es un indicador de calidad que debe ser de estricto seguimiento y ser detectado a través de los instrumentos de evaluación. Además hay que buscar estrategias para minimizar el número de estas durante la hospitalización y así poder brindar una mejor atención en salud.

De igual forma, para los cuidadores la ocurrencia de úlceras por presión fue mínimo, solo el 2.4\% los presentó. En riesgo de aparición solo se encontró que el 5,5\% lo percibieron. En la actualidad, son indicadores de calidad de atención sanitaria en pacientes críticos la incidencia de UPP reportada en la población pediátrica que varía entre el 10,2\% y el 33\% (20). Torra et al. (21) informan que las úlceras por presión son un importante problema de salud que produce daño en los pacientes y que son evitables en un alto porcentaje de casos. Es un tema que ha cobrado vigencia en la definición de políticas para el sistema de salud

En cuanto a la administración de los medicamentos, los cuidadores afirmaron que solo el $8.6 \%$ ha hecho reacción adversa y un promedio mínimo percibió riesgo de que ocurran; contrario a estos hallazgos es el estudio realizado por De las Salas et al. (22), donde se señalan que las reacciones adversas a medicamentos son comunes en niños hospitalizados y representan una morbilidad adicional y de mayor riesgo, particularmente en aquellos que usaron varios medicamentos, incluyendo antibióticos. También se indica en este estudio, realizado en dos servicios de pediatría general de dos hospitales en Barranquilla, que de 772 pacientes se detectaron un total de 156 reacciones adversas a medicamentos en 147 niños, de los cuales 138 desarrollaron una reacción adversa a medicamento durante su hospitalización y 9 niños presentaron dos. Una reacción adversa afecta de forma directa al bienestar integral del paciente, e involucra la participación de toda la institución para combatir la reacción.

De la misma manera, los cuidadores han percibido la ocurrencia de infecciones en el $2.4 \%$, y ocurrencia de 
ISSN-PRINT

1794-9831

E-ISSN 2322-7028

Vol. 16 No. 3

Sep - Dic 2019

Cúcuta, Colombia flebitis en un 17\%. El riesgo de ocurrencia en niños de flebitis fue observado en el $21,5 \%$ de cuidadores. Por su parte, Martínez et al. (10) en su análisis sobre artículos seleccionados, dedujeron que los niños son un universo aparte del resto de la población de pacientes, y como tal, tienen condiciones únicas que los hacen susceptibles a eventos adversos.

Rocco et al. (23) explican que a pesar de que hay conciencia del daño, los pacientes aún experimentan daños evitables durante sus hospitalizaciones o atenciones de salud. Lo que significa, que todavía queda mucho por hacer y hay que lograr una verdadera seguridad, lo que implica un proceso largo y puede que no sea posible eliminar por completo el daño.

Igualmente, los cuidadores percibieron que su familiar en el $17 \%$ le ha ocurrido flebitis, en cuanto al riesgo de ocurrencia el 21,5\% lo presentó. En este sentido, Buenfil et al. (24), refieren que el uso de los dispositivos intravasculares ha sido de gran utilidad clínica, ya que permite un acceso rápido y seguro al torrente sanguíneo; sin embargo, estos dispositivos no están exentos de riesgos. En este estudio realizado en pacientes hospitalizados en el Hospital de Pediatría del Centro Médico Nacional Siglo XXI, se encontró que de 410 catéteres cortos periféricos que fueron colocados a 211 niños, ningún paciente presentó flebitis infecciosa y 47 sufrieron flebitis química. Esto se debe a las buenas prácticas por parte del profesional de la enfermería, referente al cuidado del paciente y al cumplimiento de estándares y protocolos institucionales. Igual resultado encuentra Vargas et al. (15) en su estudio, quienes indican que los cuidadores perciben que los riesgos que reflejan mayor materialización son las caídas y las flebitis.

Se debe indicar, que en el servicio de pediatría en niños hospitalizados la frecuencia de eventos adversos es mayor, debido a las características particulares de esta población, por su susceptibilidad y la intensidad de las actividades que se desarrollan en este ámbito (25). Al reconocer la incidencia, los factores relacionados y las consecuencias de los errores en la atención, convierten la falla en una oportunidad de mejora, por tanto es la vía para la construcción de un sistema de salud más seguro y de calidad (26)

En consecuencia si se logra el control de los factores de riesgo relacionados con los procedimientos y/o cuidados por parte de los profesionales de la salud, se minimizaría considerablemente el riesgo de ocurrencia de EA, principalmente en servicios de alta complejidad donde se presentan mayores casos de sucesos (18).

Por su parte, los cuidadores en un $70 \%$ percibió que siempre se les informó de forma clara por parte del personal de salud sobre los riesgos y complicaciones de los tratamientos y procedimientos realizados al niño durante la hospitalización. El dato anterior se contradice frente a lo expresado por Gómez (27) quien encontró en su estudio sobre el cuidado seguro que los cuidadores en su mayoría no reciben la suficiente información sobre el concepto de catéter implantable ni por parte del médico, ni de la enfermería, lo que resulta difícil debido a la identificación del riesgo y a las medidas de prevención.

De acuerdo con lo investigado por Padilla et al. (28) las personas ven como fortalezas: el trabajo en equipo por parte de los profesionales, la disposición para la atención, la suficiencia en la información recibida y el uso de estrategias para enseñar procedimientos y aclarar dudas. Sin embargo, como dificultades comunicativas se indicaron entre otras, que el profesional haga pronósticos negativos o asuma actitudes de distancia o superioridad.

Hay que señalar, que los padres siempre están ávidos de información, valorando todos los aspectos relativos al estado de la salud del niño, así como a tratar de conocer las implicaciones que la enfermedad puede tener en su futuro. No obstante, cuando los profesionales de la salud, no tienen una perspectiva clara de aquello que los padres valorizan, reducen la posibilidad de dar una respuesta efectiva a sus necesidades, lo que contribuye a aumentar la ansiedad y la inseguridad de los padres (16).

En lo que se refiere al cuidado seguro en los procedimientos y/o pautas de prevención de riesgos y complicaciones se encontró que un $59 \%$ de la población considera que el personal de salud acude oportunamente ante sus llamados; sin embargo expresan que respecto al llamado, en procesos como UCI y cirugías no es inmediato. Al respecto, Gómez (27) cree que la comunicación, atención e información de los profesionales con los pacientes y sus cuidadores familiares para que sea eficaz, debe contener elementos tales como: escuchar con atención, explicar las cosas, dedicar tiempo suficiente e incentivar a la participación en la toma conjunta de decisiones para fortalecer la autonomía, el autocuidado y el cuidado del familiar. 
Cabe resaltar, que los entornos hospitalarios pediátricos son tan complejos que los fallos en la atención se originan inevitablemente a pesar de los esfuerzos que se hacen para detectarlos y generar una cultura de seguridad. Por ello es esencial crear o diseñar sistemas de barreras que impidan su incidencia y difundir todas aquellas intervenciones que tras la adopción de medidas han demostrado su eficacia en la reducción de errores en los hospitales pediátricos (29). En cuanto al aspecto relacionado con buscar apoyo en la detección de un posible riesgo, la mayoría de cuidadores afirmaron hacerlo siempre con un 65,6\% y 17,3\% responde que casi siempre. En sus hallazgos Cardozo et al. (30) encuentran que el personal de enfermería que se relaciona con el cuidador familiar tiene como finalidad e intención participar, colaborar y estar pendiente del cuidado del paciente durante el tiempo de hospitalización.

Al respecto la enfermería ejerce un papel muy importante para potenciar y dar a conocer las estrategias exitosas de prevención de riesgos sobre lesiones graves que afecten la seguridad del paciente. González et al. (31) manifiestan que esta temática ha sido poco documentada durante los últimos años, de ahí que sea necesario motivar a los equipos de enfermería para que reseñen sus experiencias y así darles reconocimiento a su labor realizada.

\section{Conclusiones}

- Los cuidadores percibieron seguridad en la atención pediátrica, se mantenían informados sobre riesgos, seguían las recomendaciones y conocían la razón de la hospitalización del niño. Su percepción sobre las condiciones de seguridad en la atención permitió identificar su confianza en los profesionales en situaciones inherentes a la hospitalización y en el control del riesgo.

- Predominaron los cuidadores que pertenecían al género femenino, en edades comprendidas entre 18 y 59 años, madres en unión libre, dedicadas al hogar, perteneciendo en su mayoría al estrato socioeconómico bajo.

- Los eventos adversos más frecuentes identificados por los cuidadores fueron: las flebitis, confusión de pacientes, caídas, reacción a los medicamentos y retiro accidental de dispositivos. También desconocían aspectos como exposición a riesgos y la respectiva actuación ante los mismos.

- La prestación de servicios de salud en un ambiente seguro se ha constituido en una prioridad y desafío para las instituciones en salud y los profesionales involucrados en ello. Dada la complejidad de la atención pediátrica, el presente trabajo se constituye en una investigación que sirva para mejorar los aspectos que identificaron los cuidadores, así como para obtener un cuidado seguro, realizar análisis de riesgos y reafirmar un sistema de vigilancia activo.

\section{Conflicto de intereses}

Las autoras declaran no tener ningún conflicto de interés.

\section{Referencias Bibliográficas}

1. Lima F, Souza N, Freire P, Aires de Freitas C, Bessa J, De Souza A. Implicaciones de la seguridad del paciente en la práctica del cuidado de enfermería. Enferm. glob. [Internet]. 2014[citado 2018 Ago 09]; 13(35):293-309. Disponible en: http://scielo.isciii.es/scielo.php?script=sci_arttext\&pid=S169561 412014000300017\&lng=es.

2. República de Colombia. Ministerio de Salud y Protección Social. Lineamientos para la implementación de la política de Seguridad del Paciente en la República de Colombia. Bogotá D.C, [Internet]. Noviembre de 2008. [consultado 09 de agosto de 2018]. https://www.minsalud.gov.co/Normatividad_Nuevo/Resoluci\%C3\%B2n\%200112\%20de\%202012\%20-\%20Documentos\%20de \%20 apoyo $\% 202 . p d f$

3. República de Colombia, Ministerio de la Protección Social. Guía técnica "Buena práctica para la seguridad del paciente en la atención en salud"'Internet].2014 [citado 2018 Ago 09]. Disponible en: https:/www.minsalud.gov.co/sites/rid/Lists/BibliotecaDigital/RIDE/DE/CA/Guia-buenas-practicasseguridad-paciente.pdf 
ISSN-PRINT

$1794-9831$

E-ISSN 2322-7028

Vol. 16 No. 3

Sep - Dic 2019

Cúcuta, Colombia
4. Ordoñez J. Republica de Colombia, Ministerio de protección Social, Promoción de la cultura de seguridad del paciente, Paquetes instruccionales buenas prácticas para la atención en salud. [Internet]. [citado 2018 Ago 09]. Disponible en: https://www.minsalud.gov.co/sites/rid/Lists/BibliotecaDigital/ RIDE/DE/CA/Guia_promocion_seguridad_paciente.pdf

5. De la Calle A. ¿Cómo mejorar la seguridad del paciente en una UCIP? Rev Esp Pediatr [Internet]. 2016 [Citado 09 de Agosto de 2018]; 72(1): 92-96. Disponible en: http://secip.com/wp-content/uploads/2018/05/Como-mejorar-la-seguridad-del-paciente-Y-De-la-Calle.pdf

6. Organización Panamericana de la Salud (OPS). Calidad y Seguridad del Paciente. [Internet]. [Acceso: 25 de agosto de 2018]. Disponible en: https://www.paho.org/col/index.php?option=com conten $\underline{\mathrm{t} \& \mathrm{view}=\text { article } \& \mathrm{id}=125 \text { :calidad-y-seguridad-del-paciente } \& \text { Itemid }=0}$

7. Oliveira MP, Gonçalves M, Sorgini MA. Cirugía segura en pediatría: elaboración y validación de checklist de intervenciones preoperatorias Rev.Latino-Am. Enfermagem. [Internet]. 2013 [Consultado 30 de agosto de 2018]; 21(5):1-8. Disponible en http://www.scielo.br/scielo.php?pid=S01041169 $2013000501080 \&$ script $=$ sci arttext\&tlng $=$ es

8. Delgadillo D. Satisfacción de la madre del recién nacido hospitalizado sobre la calidad del cuidado del profesional de enfermería en el Servicio de Neonatología del Instituto Nacional Materno Perinatal. Tesis de grado. Universidad Roosevelt, Lima - Perú. [Internet]. 2014 [Consultado noviembre 02 2017]; 1-62. Disponible en: http://ateneo.unmsm.edu.pe/ateneo/bitstream/123456789/4309/1/Delgadillo_Morales_Doris 2014.pdf

9. Alvarado H, Rodríguez S. Necesidades percibidas de atención por niños, cuidadores y enfermeros durante la hospitalización en una unidad de cuidado intensivo. Revista Investig Enferm. Imagen Desarr. [Internet]. 2015 [Consultado 21 de junio de 2018]; 17(1):113-130 [Consultado: noviembre 02 2017]. Disponible en: http://revistas.javeriana.edu.co/index.php/imagenydesarrollo/article/view/8665

10. Martínez C, Álvarez F, Garzón C, Rave I. Eventos adversos en niños hospitalizados: un desafío para el Cuidado de Enfermería. Rev. cienc. cuidad. [Internet]. 2019[Consultado: febrero 02 2017]; 16(1):111-123. Disponible en: https://doi.org/10.22463/17949831.1547

11. Cavalcante A, Cardoso R, Tolstenko N L, Dantas F, Santiago da Rocha S. Cuidado seguro ao paciente: contribuições da enfermagem. Revista Cubana de Enfermería [Internet]. 2015[Consultado: octubre 03 2017]; 31(4) Disponible en: http://revenfermeria.sld.cu/index.php/enf/article/view/907

12. Barrera L, Pinto N, Carrillo M, Chaparro L, Sánchez B. Cuidado a los cuidadores: familiares de personas con enfermedad crónica. Bogotá DC: Editorial de la Universidad Nacional Unibiblos, 2009.

13. Sánchez B, Carrillo M, Barrera L, Chaparro L. Carga del cuidado de la enfermedad crónica no transmisible. Revista Aquichan. [Internet]. 2013[consultado 19 de octubre de 2018]; 13(2): 247-260 Disponible en: http://aquichan.unisabana.edu.co/index.php/aquichan/article/view/2395/3228

14. República de Colombia. Ministerio de Salud. Resolución No 008430 de 1993, octubre 4, por la cual se establecen las normas científicas, técnicas y administrativas para la investigación en salud [Internet]. Santa Fe de Bogotá: El Ministerio; 1993 [consultado 29 de octubre de 2018]. Disponible en:http:// www.unisabana.edu.co/fileadmin/Documentos/Investigacion/comite de etica/Res 84301993 $\underline{\text { Salud.pdf }}$

15. Vargas E, Coral R, Moya D, Ortiz T, Mena Y, Romero E. Percepción de condiciones de seguridad del entorno hospitalario de pacientes con enfermedad crónica en Colombia. Aquichan. [Internet]. 2017[consultado 13 de octubre de 2018] ;17(1):53-69. Disponible en: http://www.scielo.org.co/pdf/ aqui/v17n1/1657-5997-aqui-17-01-00053.pdf

16. Melo EMOP, Ferreira PL, Lima RAG, Mello DF. Participación de los padres en los cuidados de la salud de niños hospitalizados. Rev. Latino-Am. Enfermagem [Internet]. 2014 [consultado 29 de octubre de 2018].;22(3):432-9 Disponible en: http://www.scielo.br/pdf/rlae/v22n3/es_0104-1169rlae-22-03-00432.pdf 
17. Alvarado H, Achury Saldaña D. Prevención de los eventos adversos en las unidades de cuidado intensivo pediátricas. Rev Salud Uninorte. [Internet]. Barranquilla (Col.) 2016. [consultado 17 de octubre de 2018]; 32 (1): 144-152. Disponible en: http://rcientificas.uninorte.edu.co/index.php/salud/article/ viewArticle/6095/8895

18. Parra CV, López JS, Bejarano $\mathrm{CH}$, Puerto AH, Galeano ML. Eventos adversos en un hospital pediátrico de tercer nivel de Bogotá. Rev. Fac. Nac. Salud Pública, [Internet]. 2017 [consultado 29 de octubre de 2018]; 35(2): 284-292. http://www.scielo.org.co/pdf/rfnsp/v35n2/0120-386X-rfnsp-35-02-00286.pdf

19. Riquelme Hernández Giselle, Ourcilleón Antognoni Astrid. Descripción de eventos adversos en un hospital pediátrico de la ciudad de Santiago, Chile. Enferm. glob. [Internet]. 2013 [consultado 17 de octubre de 2018]; 12 ( 29 ): 262-273. Disponible en: http://scielo.isciii.es/scielo.php?script=sci arttext\&pid=S1695-61412013000100013\&lng=es.

20. Frank G, Walsh KE, Wooton $S$, et al. Impact of a Pressure Injury Prevention Bundle in the Solutions for Patient Safety Network. Pediatr Qual Saf. [Internet]. 2017 [consultado 17 de octubre de 2018];(22):113.Disponible en: https://www.ncbi.nlm.nih.gov/pmc/articles/PMC6132915/

21. Torra Bou JE, Verdú Soriano JS, Lavin R, Paras Bravo P, Soldevilla Ágreda J, García Fernández FP. Las úlceras por presión como problema de seguridad del paciente. Gerokomos [Internet]. 2016 [citado 2019 Abr 10]; 27(4):161-167. Disponible en: http://scielo.isciii.es/scielo.php?script=sci arttext\&pid=S1134-928X2016000400007\&lng=es.

22. De las Salas R, Díaz D, Burgos F, Vaca C, Serrano D. Reacciones adversas a medicamentos en niños hospitalizados en Colombia. Departamento de Enfermería, Universidad del Norte, Barranquilla, Colombia. Biomimetics Laboratory, Instituto de Biotecnología Universidad Nacional de Colombia, Bogota, Colombia. [Internet]. 2016 [citado 2019 Abr 10]; 47(3):142-147. Disponible en: http://www. redalyc.org/pdf $/ 283 / 28348402003 . p d f$

23. Rocco Ch, Garrido A. Seguridad del paciente y cultura de la seguridad. Rev. Med. Clin. Condes. [Internet]. 2017 [citado 2018 Agosto 09]; 28(5) 785-795; Disponible en: https://www.elsevier.es/es-revista-revista-medica-clinica-las-condes-202-articulo-seguridad-del-paciente-y-cultura-S0716864017301268

24. Buenfil M, Espinosa G, Rodríguez R, Miranda M. Incidencia de eventos secundarios asociados al uso de catéteres cortos venosos periféricos. Rev Med Inst Mex Seguro Soc. [Internet]. 2015 [Consultado el 05 junio de 2017]; 53(3):310-315. Disponible en: http://www.medigraphic.com/pdfs/imss/im-2015/ ims1531.pdf

25. Zárate R, Olvera S, Cantoral A, Hernández S, Sánchez S et al. Factores relacionados con eventos adversos reportados por enfermería en unidades de cuidados intensivos. Proyecto multicéntrico. Enfermería universitaria [Internet]. 2015 [citado 2019 Abr 10]; 2(2): 63-72. Disponible en: http://www. scielo.org.mx/pdf/eu/v12n2/1665-7063-eu-12-02-00063.pdf

26. Silva GD, Silvino ZR, Almeida VS, Querido DL, Diaz PSC, Christovam BP. Errores de medicación en Unidades de Terapia Intensiva Neonatal. Enfermería Global. [Internet]. 2014 [consultado 29 de octubre de 2018];13(1): 370-384. Disponible: http://scielo.isciii.es/scielo.php?script=sci_arttext\&pid $=\mathrm{S} 1695-61412014000100019$

27. Gómez A. intervención educativa para el cuidado seguro del catéter implantable del niño con cáncer. [Tesis de maestría]. Bucaramanga: Universidad nacional de Colombia; 2016 [Consultado el 05 junio de 2018]. Disponible en: http://www.bdigital.unal.edu.co/55741/1/63536764.2016.pdf

28. Padilla EM, Sarmiento Medina P Ramírez Jaramillo A. Percepciones de pacientes y familiares sobre la comunicación con los profesionales de la salud. Rev. salud pública. [Internet]. 2014 [consultado 09 de Agosto de 2018];16 (4): 585-596. Disponible en: https://www.scielosp.org/article/ssm/content/ raw/?resource_ssm_path=/media/assets/rsap/v16n4/v16n4a10.pdf 
ISSN-PRINT

$1794-9831$

E-ISSN 2322-7028

Vol. 16 No. 3

Sep - Dic 2019

Cúcuta, Colombia
29. Urda Cardona AL, Peláez Cantero MJ. Seguridad hospitalaria en pediatría. Rev. Anales de Pediatría. [Internet]. 2015[Consultado el 05 junio de 2018];83(4)227-228. Disponible en: https://www.analesdepediatria.org/es-seguridad-hospitalaria-pediatria-articulo-S1695403315003355

30. Cardozo García Y, Escobar C, Cuartas Velásquez C L, Cuartas Velásquez P A. Interacción del personal de enfermería con el cuidador familiar en el ámbito hospitalario Investigaciones Andina, 2015.[Internet][consultado el 11 de Agosto de 2018]; 17(30):1224-1237. Disponible en: http://www.redalyc.org/ pdf/2390/239035878007.pdf

31. González Consuegra RV, Hernández Sandoval YT, Matiz Vera GD. Estrategias de enfermería para prevenir lesiones de piel en personas con riesgo de padecerlas. Rev Cubana Enferm [Internet]. 2017 [citado 11 Agosto 2018]; 33(3). Disponible en: http://revenfermeria.sld.cu/index.php/enf/article/view/954 